\title{
Modeling of Aerodynamic Forces on the Wind Turbine Blades
}

\author{
Asif Memon, Saleem R. Samo, M. Asad, and Fareed H. Mangi
}

\begin{abstract}
This research work is aimed to improve the wind turbine modeling for a better representation of aerodynamic forces around the blades. The modeling of forces has been carried out using the actuator surface hybrid model. This model combines the blade element method and Navier-Stokes equation solver. The forces are extracted using real airfoil section of $\mathbf{S 8 0 9}$ in order to impose on the line which represents the actuator surface. The near wake is calculated and compared with the proposed model and the existing models.
\end{abstract}

Index Terms-Aerodynamic forces, CFD, hybrid models, wind turbine wake.

\section{INTRODUCTION}

In the wind turbine park, the impact of wind speed fluctuation due to extraction of kinetic energy from the wind has a significant impact on the flow dynamics affecting the subsequent wind turbine machines downstream and thus loss of power output upto $23 \%$ of total power output in large offshore wind farms [1]-[3]. This Aerodynamic interaction, between the wind turbines installed in the wind farm, creates mainly the momentum deficit downstream which decreases the energy production due to increased mean velocity shear; and turbulence increase causes the fluctuations in dynamic loads due to unsteady flow and causing material fatigue on the subsequent wind turbines in the wake, thus decreasing the blade life.

Thereof, it is imperative to investigate the wake development downstream the wind turbine to optimize the extraction of kinetic energy from the wind. Due to computational cost and time, it's not feasible to simulate the wind turbine wake with real geometry. Simplified models have been developed to represent the flow around the wind turbines at the expense of real blade geometry representation. These simplified models are called hybrid models.

Hybrid models are used to represent the wind turbine rotors. As far, three hybrid models have been evolved: actuator disc, actuator line, and actuator surface. The models are named the way real wind turbine rotor geometry is replaced. All these modeling methods use blade element momenthum (BEM) method to calculate forces on the blades coupled with Reynolds's averaged Navier-stokes (RANS) equations with the rotor equivalent conditions to simulate flow field. Thus

Manuscript received July 14, 2014; revised September 20, 2014.

Asif Memon, Saleem R. Samo, and Fareed H. Mangi are with Quaid-e-Awam Engineering University, Pakistan (e-mail: asif.memon@quest.edu.pk).

M. Asad is with Univeristy of Management \& Technology-Lahore, Pakistan. the calculation resources are considerably reduced and the rotor may be replaced either with body forces or surface forces according to the geometry of the model. But these simplified wind turbine models like actuator disc [4]-[6], and actuator line [7] do not accurately represent the flow field around the blades and aerodynamics forces. Existing hybrid models takes into account either the pressure discontinuity [8], [9] or the source terms [10] to represent the wind turbine rotor.

In this study, an analysis on a wind turbine of NREL phase VI [11] has been carried out to measure the significance of aerodynamic tangential forces in addition to normal forces on the wind turbine blade.

\section{MESH}

As previously discussed that simplified models cannot be used with precision to represent the flow field across the wind turbine rotor necessary to evaluate the aerodynamic forces applied to blades.

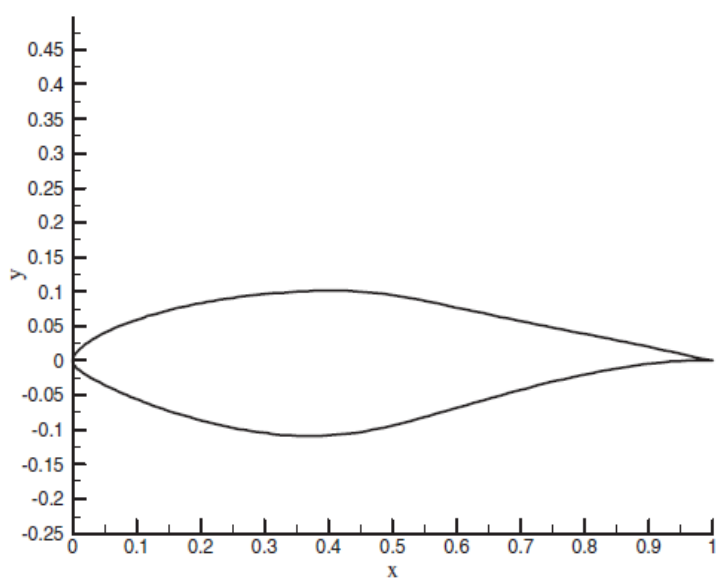

Fig. 1. Airfoil section S809 [12].

This work presents the modeling method where each blade is replaced with pressure discontinuity, lift force (normal force) and drage force (tangential force). The difficulties of body forces in the case of previous models have been overcome in this way. As the chord of the blade is varying across the blade, this has also been taken into account to resemble the real geometry of the wind turbine blade.

In order to simulate the complete geometry of S809 and hybrid modeling, the same mesh has been created and used for different models. This H-type structured mesh contains $1.10^{5}$ cells and Fig. 2, as the geometry is not complex. RANS (Reynold's averaged Navier-Stokes) has been employed and a convergence criterion has been fixed at $1.10^{-5}$. 

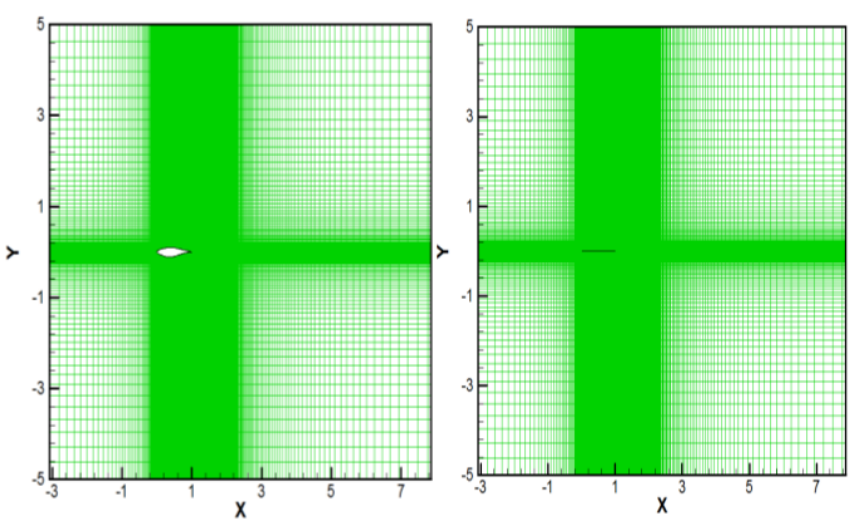

Fig. 2. Mesh of airfoil section S809 (left) and actuator surface (right).

In the RANS solver, the iterations have been pursued until lift and drag forces are established. The free stream velocity has been fixed at $10 \mathrm{~m} / \mathrm{s}$ as the incident velocity, and the turbulence model chosen is $k-\omega$ SST having turbulence intensity of $2 \%$.
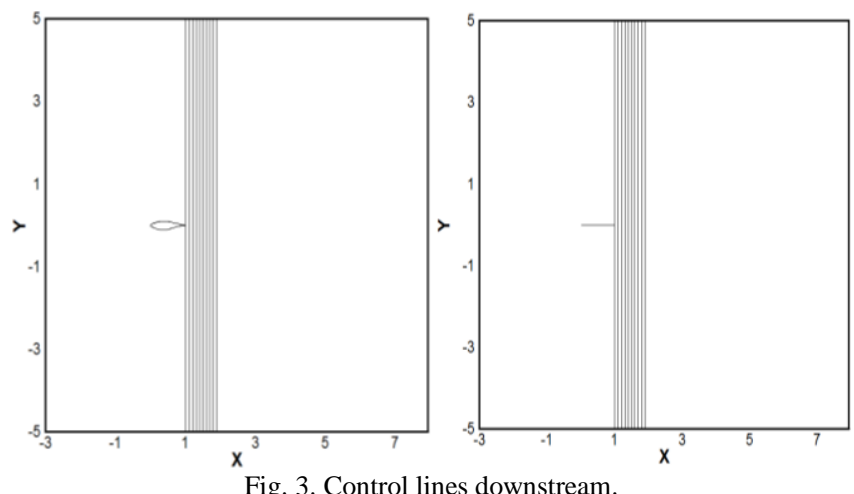

In order to visualize near wake development downstream, control surfaces are created. These control surfaces are equidistant, each having length of 0.1 chord of airfoil section, Fig. 3.

\section{VALIDATION OF SIMULATION}

The simulation of real geometry of airfoil has been carried out using the software ANSYS FLUENT 6.3 ®. The results of lift and drag coefficients of this simulation of real geometry has been compared with the experimental results of wind tunnel of Delft University [13] in order to validate the simulation of airfoil section S809.

Evolution of lift and drag coefficients with respect to angle of attack and lift coefficient with respect to drag coefficient are presented in the Fig. 4. This comparison before the stall angle shows a good agreement between the experimental and simulation results. Whereas, a slight dispersion is observed beyond the stall angle $\left(17^{\circ}\right)$ due to the fact that the flow separation is difficult to localize numerically due to transition laminar-turbulent and consequently the lift coefficient is overestimated when compared with experimental results. This dispersion beyond the stall angle may also be attributed to the limitation of $\mathrm{k}-\omega$ turbulence model in Navier-Stokes solver to reproduce separation phenomenon and estimate precisely the aerodynamic properties of an airfoil section beyond this angle.
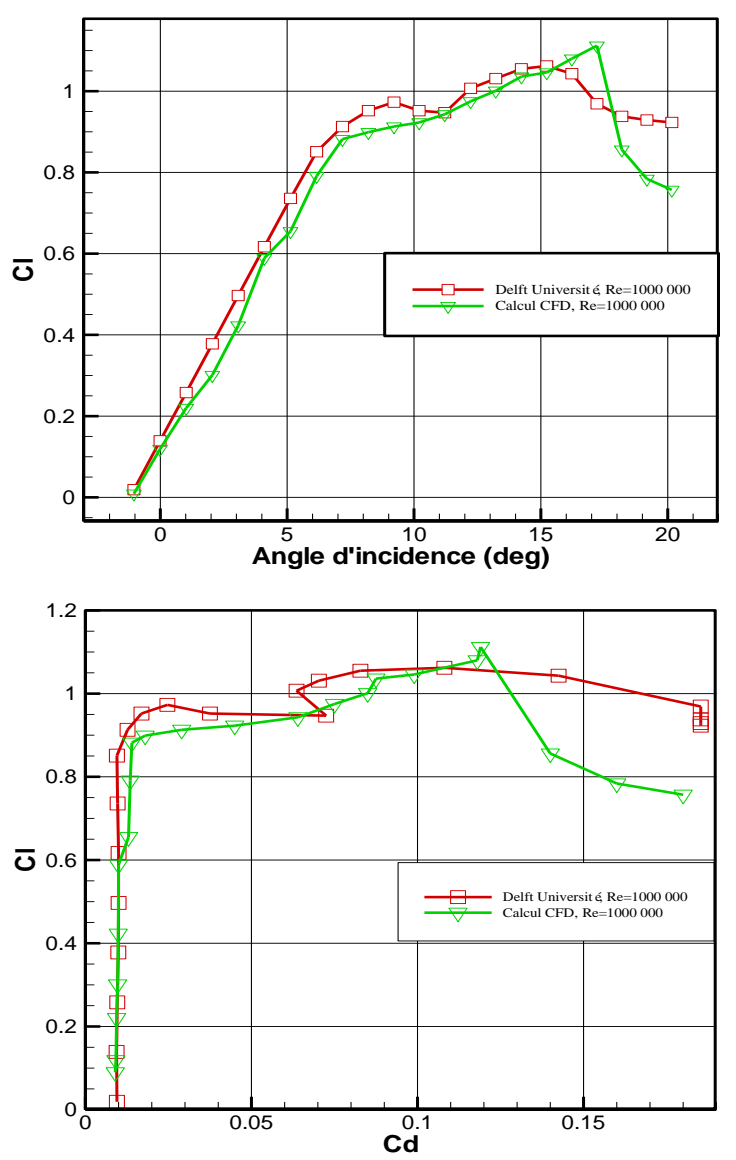

Fig. 4. Lift and drag coefficients of airfoil section S809.

\section{ANALYSIS OF NORMAL AND TANGENTIAL FORCES}

NREL Phase VI wind turbine has been studied in order to analyze experimental results. This two bladed wind turbine of $10 \mathrm{~m}$ diameter and of airfoil section of S809 has been tested in the wind tunnel of NASA at Ames [14]. These experiments contain a database for analysis of aerodynamic properties of wind turbine. This study helps to analyze the importance of tangential forces in comparison to normal forces. With the help of this study, the coefficients of tangential forces and normal forces have been calculated with respect to different angles of attack at different sections of the blade. For this, one of the blades is divided into five sections and each section represents a profile. The normal and tangential coefficients are calculated at $1.10^{6}$ Reynolds's number. Using interpolation, two bi-dimensional functions have been created $C_{n}=C_{n}(\alpha, r / R)$ and $C_{t}=C_{t}(\alpha, r / R)$.

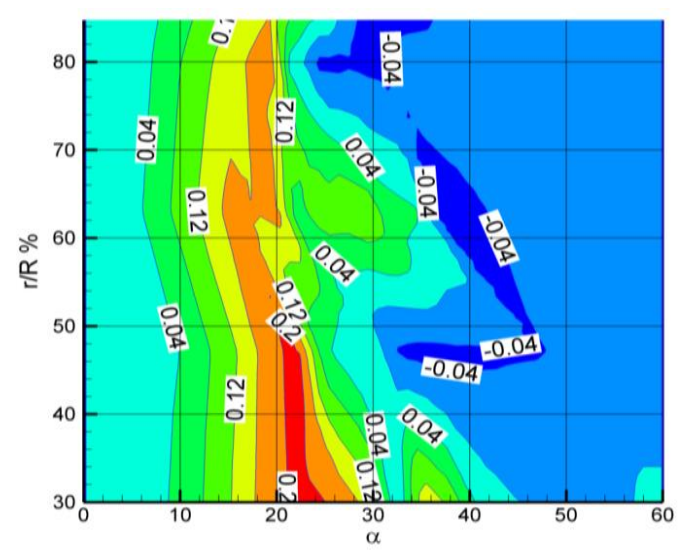




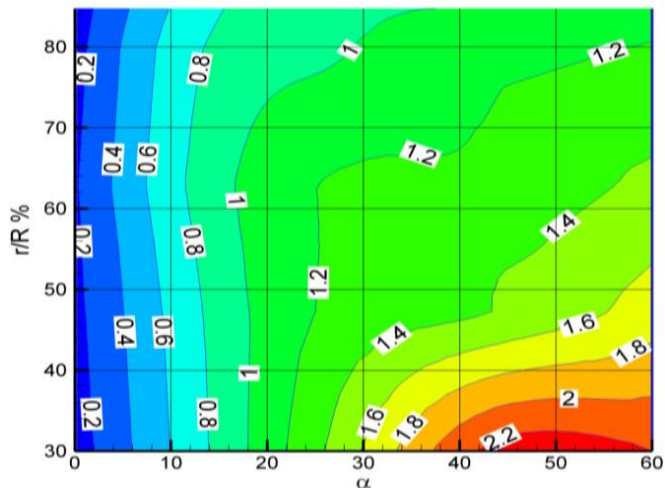

Fig. 5. (a) Normal force, (b) Tangential force.

The result of analysis is presented in Fig. 5, where $r / R$ represents each radius of the wind turbine blade tested with respect to angle of attack. The normal and tangential forces coefficient $\left(C_{n}, C_{t}\right)$ are calculated throughout the blade with respect to several angles of incidence. It can be observed that at lower angle of attacks, the coefficient of tangential forces is lower when compared with coefficient of normal forces. But, beyond $10^{\circ}$, the coefficient of tangential forces increases and becomes significant. Usually, the wind turbines operate at increased angles of attack. The analysis of this study of NREL wind turbine VI shows that tangential forces are in order of $10-20 \%$ of normal forces, Fig. 5.

\section{MATHEMATICAL MODELING}

\section{A. Aerodynamic Forces Modeling}

In proposed model, the tangential forces are taken into account in addition to normal forces in order to reproduce correctly the aerodynamic behavior using hybrid modeling. In previous models, only pressure discontinuity which represents normal forces is applied, or source terms are applied in the vicinity of hybrid model of actuator line. Here, both forces are taken into account, such that as illustrated in Fig. 6, the projection of forces in $X$ direction gives $F_{t}$ which represents tangential forces and the projection of force in $Y$ direction gives $F_{n}$ which represents normal forces.

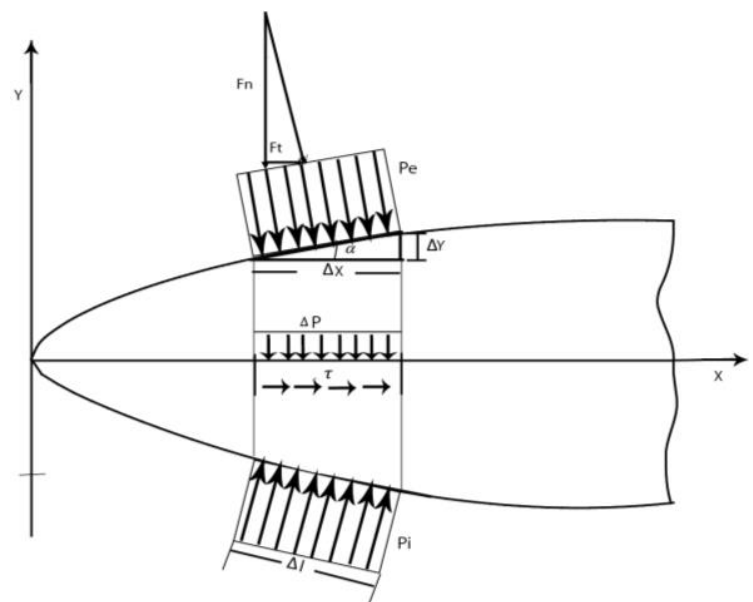

Fig. 6. Normal and Tangential Forces on airfoil section.

Considering one segment of cord $\Delta x$, it is intensity of normal and tangential forces which are applied. The components of forces in the normal and axial directions respectively are:

$$
\begin{aligned}
& F_{n}=F \cos \alpha=p \Delta l \cos \alpha=p \Delta X \\
& F_{t}=F \cos \alpha=p \Delta l \sin \alpha=p \Delta Y
\end{aligned}
$$

$\Delta l$ represents length of suction side and corresponding $\Delta X$. The normal force on the cord is applied as

$$
F_{n}=\left(p_{i}-p_{e}\right) \Delta X
$$

where $p_{i}$ is pressure on the pressure side, and $p_{e}$ is the pressure on the suction side. And the intensity of normal forces on the cord is applied as:

$$
\Delta p=\frac{F_{n}}{\Delta X}=\left(p_{i}-p_{e}\right)
$$

The total tangential forces on the cord are calculated as the difference between forces on the suction side and on the pressure side. Whereas indices $i$ and $e$ are designated respectively for pressure side and suction side, tangential forces can be written as:

$$
\tau=p_{e} \cdot \mathrm{t}_{g}\left(\alpha_{e}\right)+p_{i} \cdot \mathrm{t}_{g}\left(\alpha_{i}\right)
$$

These forces are applied with the help of sub-programme called UDF (user defined function) and coupled with the solver of Navier-Stokes equations. To reproduce the tangential forces, in this proposed model, these forces are represented by sources terms at the vicinity of a line of pressure discontinuity; and flow field is simulated by the solver of Navier-Stokes equation.

\section{B. Hybrid Modeling}

To model the flow with the help of actuator line, the aerodynamic forces are extracted from the results of complete simulation of airfoil section S809. In the domain of calculation of hybrid model, the airfoil is replaced with a circle centered at $1 / 4$ of the cord of the airfoil. The forces then are distributed in the circle as source terms, for this, a sub program is integrated in the CFD solver in order to distribute these forces. The normal and tangential forces by the airfoil on the flow are:

$$
\begin{aligned}
& d f_{t}=\frac{1}{2} \rho V^{2} C_{t} d r \\
& d f_{n}=\frac{1}{2} \rho V^{2} C_{n} d r
\end{aligned}
$$

Whereas, $C_{t}$ and $C_{n}$ are respectively the coefficients of tangential forces and normal forces. Jourieh has elaborated the choice of diameter to represent the blade and deducted that better results can be obtained when the circle diameter is between 0.3 and 0.6 of the cord. Actuator line model in this case gives the model of actuator cylinder with uniform distribution of source terms on each section. In this modeling, 0.3 times the cord has been chosen.

In modeling of actuator surface with pressure discontinuity, the aerodynamic forces on the pressure side and on the suction 
side are extracted from the simulation results of airfoil section S809. Then, the airfoil is replaced with a line which represent actuator surface which coincides with the cord of airfoil. This model of actuator line corresponds to 'pressure discontinuity'. The pressure discontinuity is imposed on line as $\Delta p=p^{+}-p^{-}$it is actually a boundary condition of 'fan' imposed with the help of sub program integrated in the Navier-Stokes solver. As the line coincides with cord so the condition to impose the pressure discontinuity is $0 \leq c \leq 1$. When this condition is satisfied, the Navier-Stokes solver simulates the flow field.

\section{Simulation Results}

\section{A. Flow Field}

In order to validate the proposed model, the simulation has been carried out with the existing model for the purpose of comparison. The calculation results of flow field at different angles of attack are compared. Following comparison is carried out:

1) Complete geometry of airfoil section as reference

2) Actuator surface model with pressure discontinuity and source terms

3) Actuaotr surface model only with pressure dicontinuity

4) Actuator line model with source terms

These results show the best reproduction of field with the help of model proposed has been produced when the together the pressure discontinuity and source terms has been applied on actuator surface. And, existing model of actuator line with only source terms and actuator surface with pressure discontinuity do not correctly reproduce the flow field.

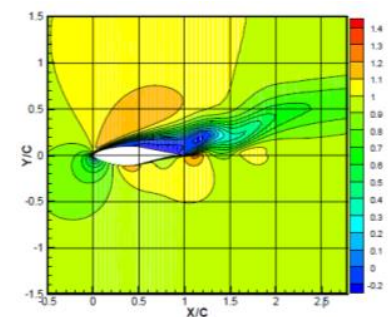

(a)

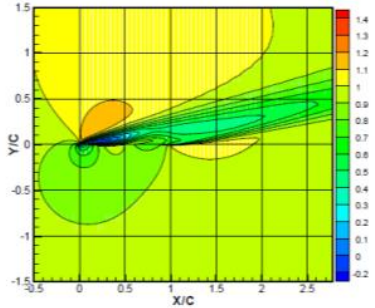

(c)

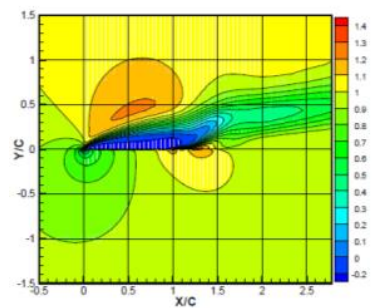

(b)

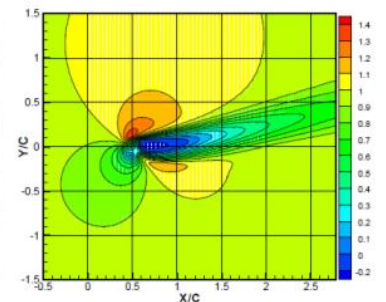

(d)
Fig. 7. Flow field around (a) airfoil section S809 (b) proposed model (c) actuator surface (d) actuator line.

The velocity gradient is also correctly reproduced not only around the geometry but also at the leading edge and trailing edge; whereas, actuator line is far from the reality. The proposed model presents the coherent flow field as that of real geometry of S809. Notably, the flow deceleration is also well reproduced and flow separation. This model can be applied on the wind turbine modeling at several angles of attack.

\section{B. Near Wake}

A comparison concerning near wake development has been presented in Fig. 8; the comparison is shown among the results of real geometry of S809 with the hybrid modeling. The case of real geometry is designated as 'S809' which serves as the reference, the second case designated ' $\Delta p+\Delta \tau$ ' represents the results of the proposed model with pressure discontinuity and source terms/tangential forces imposed in the vicinity of the actuator surface, the third case is of only pressure discontinuity $\Delta p$ which is imposed on the actuator surface, and the fourth case is presented with the distribution of only source terms/volume forces on $1 / 4$ of the cord.
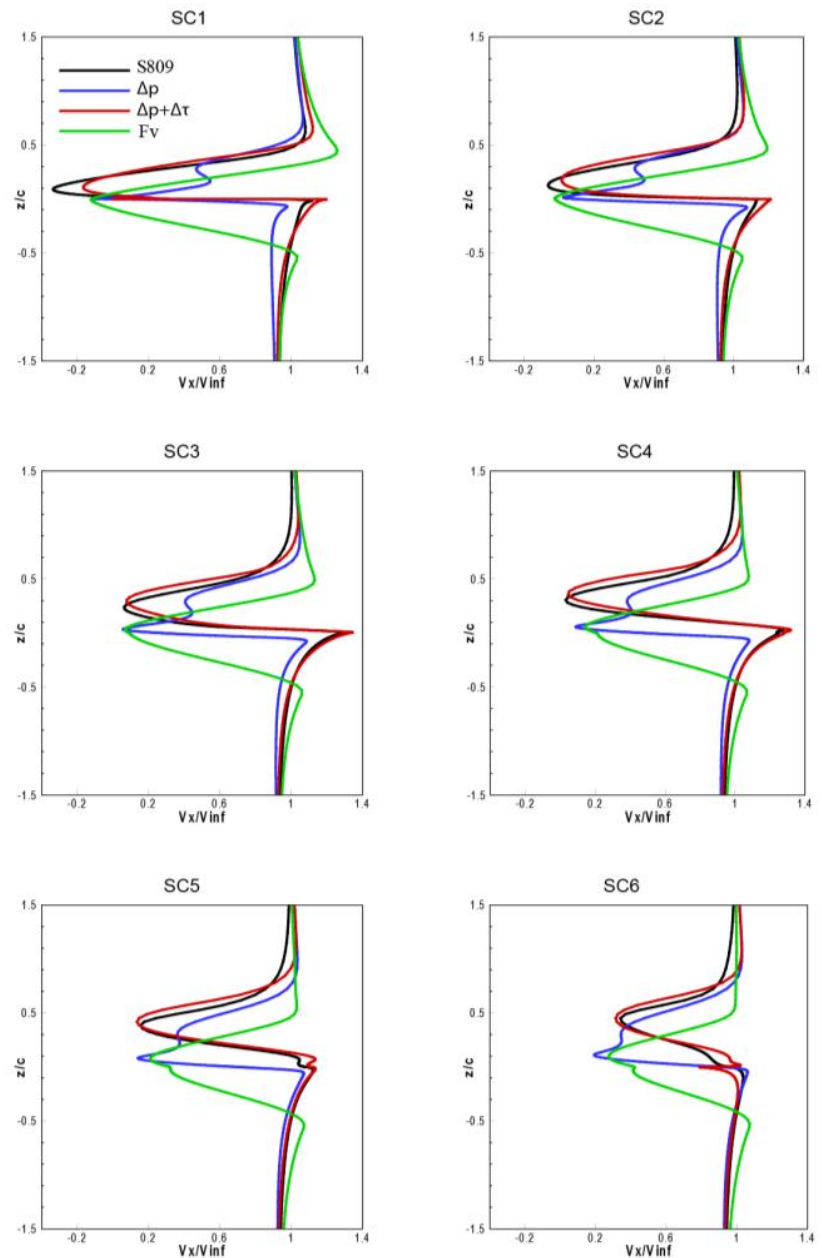

Fig. 8. Near wake development at control surface.

To clearly visualize the near wake, ten control lines are drawn and designated as CS1 for control line one, and CS6 for control line sixth. These control lines are equally distant of 0.1Cord from each other which are created downstream.

The comparison of near wake shows that the wake calculated with the help of proposed model is more coherent with that of complete geometry of S809. Whereas, other two model shows a shift which is non-negligible. This may be noted here that at important angles of attack, the influence of tangential forces becomes significant. Finally, the model of actuator cylinder is presented with distribution of source terms on every section gives the results incoherent. It may be noted that this near wake has been calculated at 15 , where the 
separation starts, and even then the proposed model is comparable with the real geometry. Whereas, other existing models are far away from reality.

\section{CONCLUSION}

The modeling of aerodynamic forces on wind turbine blades has been carried out by taking into accounts both the normal forces and tangential forces. The normal forces are imposed as pressure discontinuity on the geometry which represents the wind turbine blades, and normal forces are imposed as source terms in the vicinity of blades. This improved technique has shown significant improvement in order to represent the flow field around the airfoil section and in near wake. The results obtained using normal and tangential forces are satisfactory when compared with the simulation results of real geometry of wind turbine blades. Further work may be carried out with variable airfoil thickness along the blades.

\section{REFERENCES}

[1] L. J. Vermeera, J. N. Sorensen, and A. Crespo, "Wind turbine wake aerodynamics," Progress in Aerospace Sciences, vol. 39, pp. 467-510, 2003.

[2] B. Lange, H.-P. Waldl, R. Barthelmie, A. G. Guerrero, and D. Heinemann, "Modelling of offshore wind turbine wakes with the wind farm program FLaP," Journal of Wind Energy, vol. 6, issue 1, pp. 87-104, 2003.

[3] W. Z. Shen, J. N. Sørensen, and R. Mikkelsen, "Tip loss correction for actuator/navier-stokes computations," J. Sol. Energy Eng., vol. 127, no. 2, pp. 209-213, April, 2005.

[4] A. Crespo, J. Hernández, and S. Frandsen, "Survey of modelling methods for wind turbine wakes and wind farms," Wind Energy, vol. 2, pp. 1-24, 1998.

[5] D. J. A. Sharpe, "General momentum theory applied to an energy extracting actuator disc," Journal of Wind Energy, vol. 7, no. 3, pp. 177-188, 2004.

[6] R. Mikkelsen, "Actuator disc methods applied to wind turbines," $\mathrm{PhD}$ Thesis, Technical University Denmark, p. 121, 2003.

[7] N. Troldborg, "Actuator line modeling of wind turbine wakes," $\mathrm{PhD}$ Thesis, Technical University of Denmark, 2008.

[8] C. S. Watters and C. Masson, "Modeling of lifting-device aerodynamics using the actuator surface concept," International Journal for Numerical Methods in Fluids, vol. 62, no. 11, pp. 1264-1298, 2009.

[9] I. Dobrev, "Modèle hybride de surface active pour l'analyse du comportement aérodynamique des rotors éoliens à pales rigides ou déformables," PhD Thesis, Arts et Métiers ParisTech, France, 2009.

[10] W. Z. Shen, J. N. Sorensen, and J. H. Zhang, "Actuator surface model for wind turbine flow computations," Proceedings of EWEC, Milan, Italy, 2007.
[11] D. Simms, S. Schreck, M. Hand, and L. Fingersh, "NREL unsteady aerodynamics experiment in the NASA-Ames wind tunnel: A comparison of predictions to measurements," Tech. Rep. NREL/TP-500-29494, National Renewable Energy Laboratories, 2001.

[12] D. Sommers, "Design and experimental results for S809 airfoil," National Renewable Energy Lab, USA, 1994.

[13] J. N. Van Ingen, L. M. M. Boermans, and J. J. H. Blom, "Low speed airfoil section research at Delft University of Technology" in Proc. International Council of the Aeronautical Sciences, 1981, pp. 401-416.

[14] M. Hand, D. Simms, and L. J. Fingersh, "Unsteady aerodynamics experiment phase VI: wind tunnel test configurations and available data campaigns,” Tech. Rep. NREL/TP-500-29955, 2001.

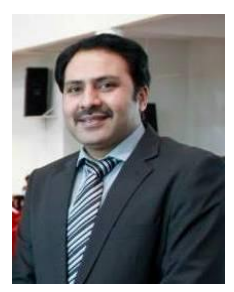

Asif Memon received his master degree in renewable energies from Corsica University in 2008 and earned his doctorate degree on wind turbine technology from Arts et Metiers ParisTech, France in 2012. He is currently an associate professor and the head of Energy and Environment Deparment, Quaid-e-Awam Engineering University, Pakistan. His research interests include renewable energies, computational fluid dynamics, and energy storage. He has more than 10 research publications on his credit.

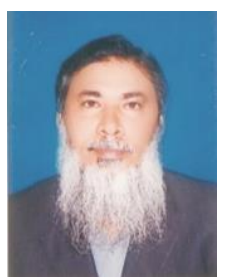

Saleem Raza Samo graduated from Asian Institute of Technology (Thailand) and UMIST (UK) in 1999. He is a professor at the Department of Energy and Environment Engineering, and the vice chancellor of Quaid-e-Awam Engineering University, Pakistan. He is specializes in renewable energies and energy efficiency in buildings. He is an author/co-author of more than 60 publications.

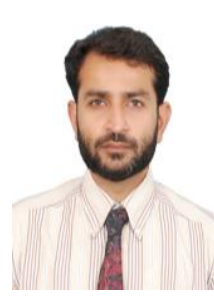

Muhammad Asad is a graduate of UET-Lahore, Pakistan \& INSA-Lyon, France. He is currently working as an associate professor and the chair of Mechanical Engineering Department, Univeristy of Management \&Technology-Lahore, Pakistan. He has rich and diversified experience R \& D and Academia. Modeling \& Simulation of Materials \& Processes are the areas of his research interest. $\mathrm{He}$ is author/co-author of over 24 scholarly articles.

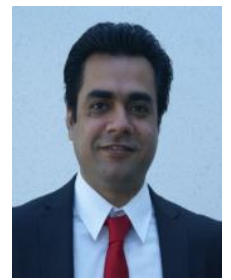

Fareed Hussain Mangi is a graduate of Polytechnic School of University of Nantes, France in the field of process intensification in energy systems. $\mathrm{He}$ is currently working as an assistant professor in Energy and Environment Engineering Department of Quaid-e-Awam University of Engineering, Science and Technology Nawabshah. He has an experience of over 10 years in the field of teaching and industrial sector. 